\title{
Surgical management of primary colonic lymphoma: Big data for a rare problem
}

\author{
Lillias H. Maguire $\mathrm{MD}^{1}{ }^{1}$ | Timothy M. Geiger $\mathrm{MD}^{2}$ | Karin M. Hardiman $\mathrm{MD}^{1}$ (0) | \\ Scott E. Regenbogen MD MPH ${ }^{1}$ | Michael Benjamin Hopkins MD $^{2}$ | \\ Roberta L. Muldoon $\mathrm{MD}^{2} \mid$ Molly M. Ford $\mathrm{MD}^{2}$ | Alexander T. Hawkins MD, MPH${ }^{2} \odot$
}

${ }^{1}$ Division of Colorectal Surgery, Department of Surgery, University of Michigan, Ann Arbor, Michigan

${ }^{2}$ Section of Colon \& Rectal Surgery, Department of Surgery, Vanderbilt University Medical Center, Nashville, Tennessee

\section{Correspondence}

Alexander T. Hawkins, MD, MPH, Section of Colon \& Rectal Surgery, Vanderbilt University, 1161 21st Ave South, Room D5248 MCN,

Nashville, TN 37232

Email: alex.hawkins@vumc.org

\begin{abstract}
Background and Objectives: Primary colonic lymphoma ( $P C L)$ is rare, heterogeneous, and presents a therapeutic challenge for surgeons. Optimal treatment strategies are difficult to standardize, leading to variation in therapy. Our objective was to describe the patient characteristics, short-term outcomes, and five-year survival of patients undergoing nonpalliative surgery for PCL.

Methods: We performed a retrospective cohort analysis in the National Cancer Database. Included patients underwent surgery for PCL between 2004 to 2014 . Patients with metastases and palliative operations were excluded. Univariate predictors of overall survival were analyzed using multivariable Cox proportional hazard analysis.

Results: We identified 2153 patients. Median patient age was 68. Diffuse large B-cell lymphoma accounted for $57 \%$ of tumors. 30- and 90-Day mortality were high $(5.6 \%$ and $11.1 \%$, respectively). Thirty-nine percent of patients received adjuvant chemotherapy. For patients surviving 90 days, 5 -year survival was $71.8 \%$. Chemotherapy improved survival (surgery+chemo, $75.4 \%$ vs surgery, $68.6 \%$; $P=.01$ ). Adjuvant chemotherapy was associated with overall survival after controlling for age, comorbidity, and lymphoma subtype (HR 1.27; $95 \% \mathrm{Cl}, 1.07-1.51 ; P=.01)$. Conclusions: Patients undergoing surgery for $\mathrm{PCL}$ have high rates of margin positivity and high short-term mortality. Chemotherapy improves survival, but $<50 \%$ receive it. These data suggest the opportunity for improvement of care in patients with PCL.
\end{abstract}

\section{KEYWORDS}

colorectal surgery, extranodal lymphoma, primary gastrointestinal lymphoma

\section{1 | INTRODUCTION}

Primary gastrointestinal (GI) lymphoma is a rare heterogeneous malignancy arising in the $\mathrm{GI}$ tract as a primary extranodal tumor. The colorectum accounts for $6 \%$ to $12 \%$ of Gl lymphoma, but among colonic neoplasms primary colonic lymphoma $(\mathrm{PCL})$ is a rare entity: representing $<1 \%$ of all colorectal tumors. ${ }^{1}$ The vast majority of $\mathrm{PCL}$ arises from the B-cell lineage, but as they may arise at any stage of maturation of T-cells, B-cells, or Natural killer (NK) cells, there exists a large number of potential histologic subtypes. ${ }^{2}$ Treatment of lymphoma of the colon or rectum may include chemotherapy, radiation, and surgery.

The outcomes of, and indications for, surgical resection in colorectal lymphoma remain unclear. Multi-institutional studies indicate $48 \%$ to $61 \%$ of patients with PCL have surgery. ${ }^{3-5}$ Surgery provides a definitive diagnosis, palliates symptoms, may prevent 
tumor perforation on chemotherapy, provides local control for aggressive disease, and may be the definitive treatment for chemotherapy-resistant indolent histologic subtypes. However, surgery is morbid, does not treat systemic disease, and does not definitively improve survival. ${ }^{6,7}$ Due to the rarity of $\mathrm{PCL}$, the bulk of the available surgical data is retrospective and combines multiple GI sites, pathologic stages, and histologic subtypes. ${ }^{3,4}$ Therefore, when a surgical approach, elective or urgent, is chosen for a PCL patient, there is a knowledge gap in the expected short and long term outcome of the patient.

The National Cancer Database (NCDB) captures approximately $70 \%$ of newly diagnosed cancers in the United States and demonstrates concordance with most population-based registries. ${ }^{8}$ Therefore, the NCDB is a platform for interrogating "real world" outcomes of surgical patients with PCL. The purpose of this study is to provide a thorough, modern, multi-institutional description of the short and long terms outcomes of patients undergoing nonpalliative surgical treatment of PCL to inform treatment decisions and patient counseling. We hypothesize that the addition of adjuvant chemotherapy will be associated with increased survival.

\section{2 | METHODS}

\section{1 | Data source}

Data for this study was extracted from the Participant User File of the NCDB. The NCDB is a joint project between the American College of Surgeons' Commission on Cancer ( $\mathrm{CoC}$ ) and the American Cancer Society. ${ }^{9,10}$ The NCDB gathers data from more than 1700 CoC-accredited centers across the United States and Puerto Rico. The NCDB is estimated to capture approximately $70 \%$ of new cancer diagnoses and now contains more than 30 million patient files.

\section{2 | Study design}

This project was reviewed and deemed exempt by the Vanderbilt University Medical Center Institutional Review Board (Protocol 161707). We conducted a retrospective, cohort analysis of a prospectively maintained database. We included all adult patients in the NCDB from 2004 to 2014 with a diagnosis of PCL (International Classification of Diseases for Oncology, 3rd Edition SEER Topography Codes C180-2, C184, C186-9, C199 with Histology Code in Appendix 1). Exclusion criteria included stage IV disease, patients operated on for palliative indications (as defined in the NCDB), and surgery performed outside the reporting facility. The primary exposure was the addition of adjuvant chemotherapy to surgical resection, as defined by the NCDB.

\section{3 | Outcomes}

The primary outcome of this study was overall survival. Due to the high rate of 90-day mortality, comparisons were made only for patients that survived 90 days. Secondary outcomes included RO resection, 30 and 90-day mortality. The NCDB does not capture disease-free survival.

\section{4 | Covariates}

Patient-level variables included age, sex, race, and education and income as markers of socioeconomic status. Comorbidities are captured by the NCDB's modification of the Charlson score, which excludes the primary cancer diagnosis condition and then reports the Charlson score as 0 (no comorbidities), 1 (total Charlson score of 1 ) or 2 (total Charlson score of 2 or more). Hospital-level data collected included the cancer designation of the hospital where the surgery was performed (community cancer program, comprehensive community cancer program, academic/research program). Type of PCL was also determined using the International Classification of Diseases for Oncology, 3rd Edition SEER Histology code. (Appendix 1). For the survival analysis, marginal and follicular lymphoma were combined to form a single category.

\section{5 | Statistical analysis}

Continuous and categorical data are expressed as mean with standard deviation and proportions, with the exception of continuous variables with grossly skewed distributions that are reported as the median with the interquartile range. Continuous and categorical variables were compared with the Student $t$ test and the $\chi^{2}$ test, respectively. For the survival analysis, the log-rank test was used for

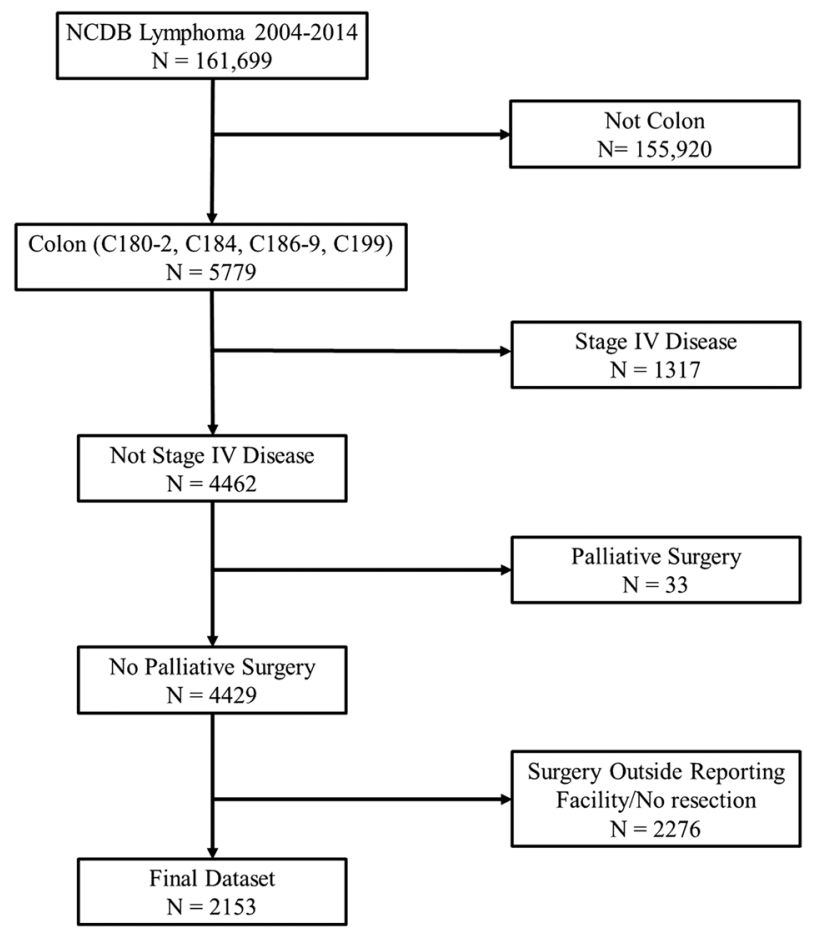

FIGURE 1 Flowchart of inclusion and exclusion criteria from the National Cancer Database (NCDB) 
TABLE 1 Patient characteristics grouped by treatment with surgery alone $(n=1286)$ vs surgery with neoadjuvant or adjuvant chemotherapy $(n=867)$

\begin{tabular}{|c|c|c|c|c|}
\hline & All $(n=2153)$ & $\begin{array}{l}\text { Surgery alone } \\
(n=1286)\end{array}$ & $\begin{array}{l}\text { Surgery+chemo } \\
(\mathrm{n}=867)\end{array}$ & $P$ value \\
\hline Age, median, y (IQR) & $68(56-78)$ & $71(59-81)$ & $64(52-74)$ & $<.001$ \\
\hline Race & & & & 0.70 \\
\hline White & 1895 (88.0\%) & 1130 (87.9\%) & 765 (88.2\%) & \\
\hline Insurance type & & & & $<.001$ \\
\hline Private & 784 (36.4\%) & 416 (32.3\%) & $368(42.4 \%)$ & \\
\hline Medicare & 1155 (53.6\%) & 751 (58.4\%) & 404 (46.6\%) & \\
\hline Medicaid & 91 (4.2\%) & 49 (3.8\%) & 42 (4.8\%) & \\
\hline$<\$ 38000$ & 254 (12.2\%) & 215 (16.9\%) & 132 (15.5\%) & \\
\hline$\$ 38000-\$ 47999$ & 397 (19.1\%) & $308(24.3 \%)$ & 212 (24.8\%) & \\
\hline$\$ 48000-62999$ & 565 (27.3\%) & 351 (27.7\%) & 219 (25.6\%) & \\
\hline$>\$ 63000$ & 857 (41.3\%) & 394 (31.1\%) & 291 (34.1\%) & \\
\hline Low education ( $>21 \%$ of adults not graduating from high school) & $331(15.4 \%)$ & 203 (15.8\%) & $128(14.7 \%)$ & .51 \\
\hline Urban-rural continuum & & & & .79 \\
\hline Metro & 1699 (78.9\%) & 1015 (78.9\%) & $684(78.9 \%)$ & \\
\hline Urban & $412(19.1 \%)$ & $23(1.8 \%)$ & $19(2.2 \%)$ & \\
\hline Facility & & & & .02 \\
\hline Community cancer program & $635(29.5 \%)$ & $354(27.5 \%)$ & $281(32.4 \%)$ & \\
\hline Comprehensive community cancer program & 975 (45.3\%) & 609 (47.4\%) & $366(42.2 \%)$ & \\
\hline Academic/research program & $543(25.2 \%)$ & $323(25.1 \%)$ & $220(25.4 \%)$ & \\
\hline Lymphoma subtype & & & & $<.01$ \\
\hline DLBCL & $1228(57.0 \%)$ & $655(50.9 \%)$ & 573 (66.1\%) & \\
\hline Burkitt & $143(6.6 \%)$ & $56(4.3 \%)$ & 87 (10.0\%) & \\
\hline Follicular & $155(7.2 \%)$ & 107 (8.3\%) & $48(5.5 \%)$ & \\
\hline Hodgkin & 45 (2.1\%) & $34(2.6 \%)$ & $11(1.3 \%)$ & \\
\hline Mantle Cell & 76 (3.5\%) & 48 (3.7\%) & $28(3.2 \%)$ & \\
\hline Marginal & 243 (11.3\%) & 216 (16.8\%) & 27 (3.1\%) & \\
\hline NHL NOS & $110(4.7 \%)$ & $62(4.8 \%)$ & 48 (5.5\%) & \\
\hline T-cell & $51(2.4 \%)$ & 32 (2.5\%) & $26(3.0 \%)$ & \\
\hline Other & $102(4.7 \%)$ & 76 (5.9\%) & $19(2.2 \%)$ & \\
\hline
\end{tabular}

Abbreviations: DLBCL, diffuse large B-cell lymphoma; IQR, interquartile range; NHL, non-Hodgkin lymphoma; NOS, not otherwise specified. 


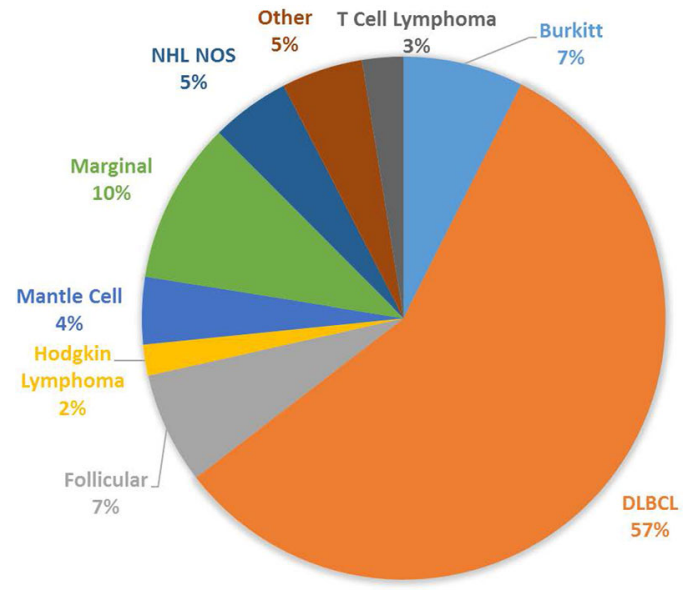

FIGURE 2 Distribution of primary colonic lymphoma histologic subtype. DLBCL, diffuse large B-cell lymphoma; NHL, non-Hodgkin lymphoma; NOS, not otherwise specified [Color figure can be viewed at wileyonlinelibrary.com]

bivariate comparison. All factors found to be significant for overall survival in a bivariate analysis were compared using a multivariable Cox proportional hazard model. All variables were initially considered using a backward Wald stepwise procedure with a $P$ value of .20 to enter and .05 to eliminate variables. SAS statistical software (version 9.3; SAS Institutes Inc, Cary NC) was used for all analyses. All tests were two-sided with an alpha level of .05 .

\section{3 | RESULTS}

\section{1 | Exposure and demographics}

After applying exclusion criteria, a total of 2153 patients with $\mathrm{PCL}$ who underwent surgery were identified in the NCDB from 2004 to 2014 (Figure $1 \&$ Table 1). The median age was 68 (interquartile range, 56-78) and 769 (35.7\%) were female. A total of 867 patients (40.2\%) underwent both surgery and adjuvant chemotherapy. The

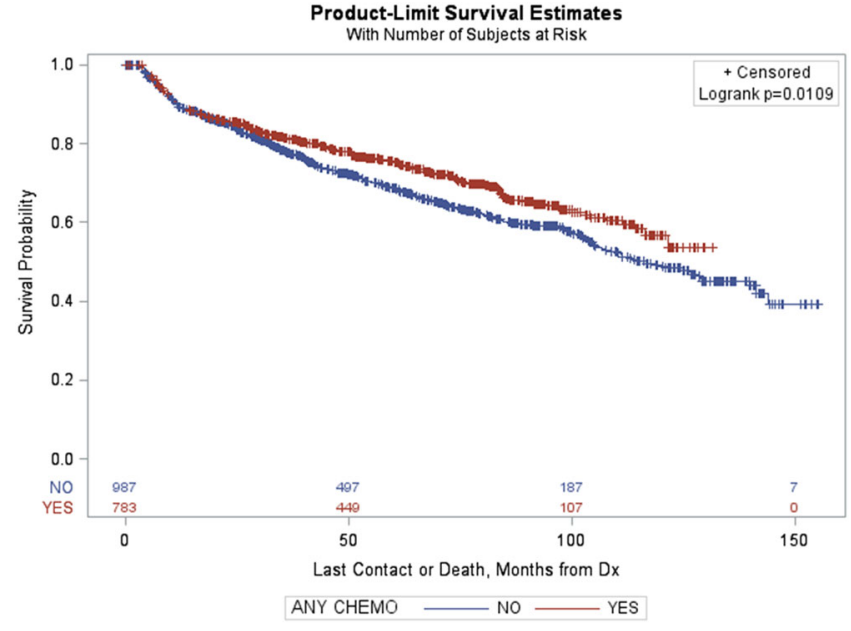

FIGURE 3 Kaplan-Meier curve for overall survival stratified by adjuvant chemotherapy [Color figure can be viewed at wileyonlinelibrary.com]

majority of patients had a diffuse large B-cell lymphoma (DLBCL) subtype (57.0\%) with marginal (10.3\%) and follicular (7.2\%) comprising the top three subtypes. (Figure 2) Factors associated with the use of adjuvant chemotherapy included younger age, male sex, private insurance, treatment at a community cancer program, and DLBCL subtype.

\section{2 | Perioperative outcomes}

Preoperative chemo- and radiotherapy were rarely administered in this cohort, suggesting that the identified group of patients represent a "surgery first" approach rather than treatment failures. (Table 2). Overall, rates of RO resection were relatively low (54.2\%) and were modestly higher in the surgery-only cohort (surgery+chemo $50.7 \%$ vs surgery $56.5 \%$; $P=.008$ ). Postoperative radiation was rarely administered ( $1.8 \%$ total). Rates of both 30 (5.6\%) and 90-day (11.1\%) mortality were high. To further

TABLE 2 Treatment characteristics, short-term outcomes, and overall survival of patients treated with surgery alone $(\mathrm{n}=1286)$ vs with surgery and chemotherapy $(n=867)$

\begin{tabular}{|c|c|c|c|c|}
\hline & All $(n=2153)$ & Surgery alone $(n=1286)$ & Surgery+chemo $(n=867)$ & $P$ value \\
\hline Preoperative chemotherapy & $33(1.5 \%)$ & N/A & $33(3.8 \%)$ & \\
\hline Preoperative radiation & $5(0.2 \%)$ & $1(0.1 \%)$ & $4(0.4 \%)$ & .07 \\
\hline Length of stay, $d$ median (IQR) & $6(4-9)$ & $6(4-9)$ & $6(4-8)$ & .01 \\
\hline Postoperative chemotherapy & $840(39.0 \%)$ & N/A & $840(96.9 \%)$ & \\
\hline 30-d readmission & $143(6.6 \%)$ & $90(7.0 \%)$ & $53(6.1 \%)$ & .41 \\
\hline 30-d mortality & $121(5.6 \%)$ & $108(9.1 \%)$ & $13(1.6 \%)$ & $<.001$ \\
\hline 90-d mortality & $238(11.1 \%)$ & $197(16.6 \%)$ & $41(5.0 \%)$ & $<.001$ \\
\hline 5-yr overall survival (patients surviving $90 \mathrm{~d}$ ) & $71.8 \%$ & $68.6 \%$ & $75.4 \%$ & .01 \\
\hline
\end{tabular}

Abbreviation: IQR, interquartile range. 
TABLE 3 Results of bivariate and multivariable Cox proportional hazards model analysis of patient, treatment, and tumor factors associated with overall survival among 2153 primary colonic lymphoma patients

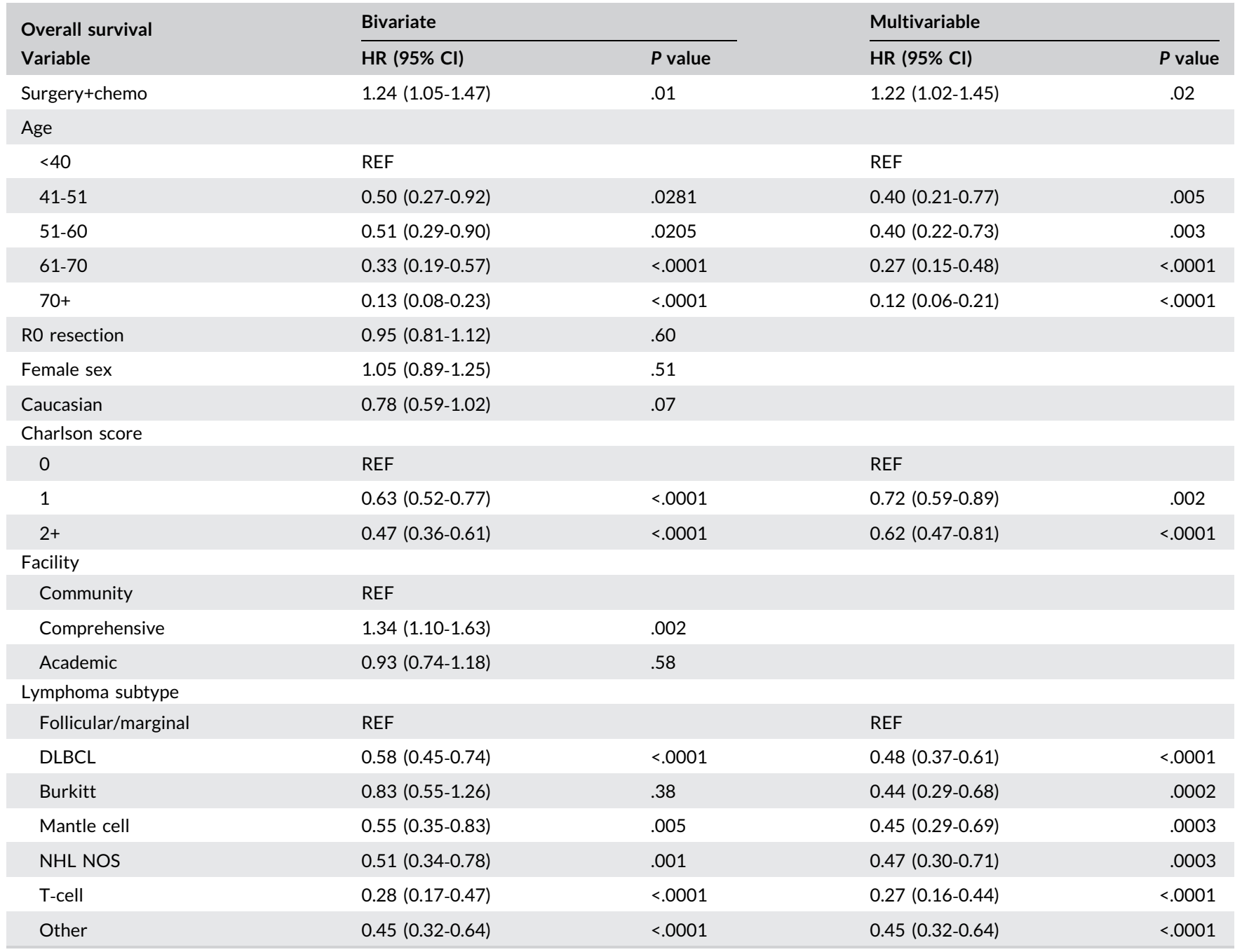

Abbreviations: DLBCL, diffuse large B-cell lymphoma; HR: hazard ratio; NHL, non-Hodgkin lymphoma; NOS: not otherwise specified.

investigate the role of surgery in long term outcomes of $\mathrm{PCL}$ patients, perioperative deaths were excluded.

\section{3 | Overall survival}

A total of 1915 (88.9\%) patients survived 90 days and were included in the analysis of overall survival. Over a mean follow up of 53 months, the 5 -year overall survival for the cohort was $71.8 \%$. Thirty-nine percent of patients received adjuvant chemotherapy. The administration of adjuvant chemotherapy was associated with improved 5 -year overall survival (surgery + chemo $75.4 \% \%$ vs surgery $68.6 \% ; P=.01$; Figure 3 ) This relationship remained in a multivariate Cox proportional hazard analysis that included age, Charlson comorbidity score and lymphoma subtype (HR 1.22; 95\% Cl, 1.02-1.45; $P=.02$; Table 3). RO resection was not associated with overall survival. An analysis of overall survival by lymphoma subtype showed follicular/marginal lymphoma to be associated with the best survival and T-cell lymphoma to be associated with the worst; Figure 4).

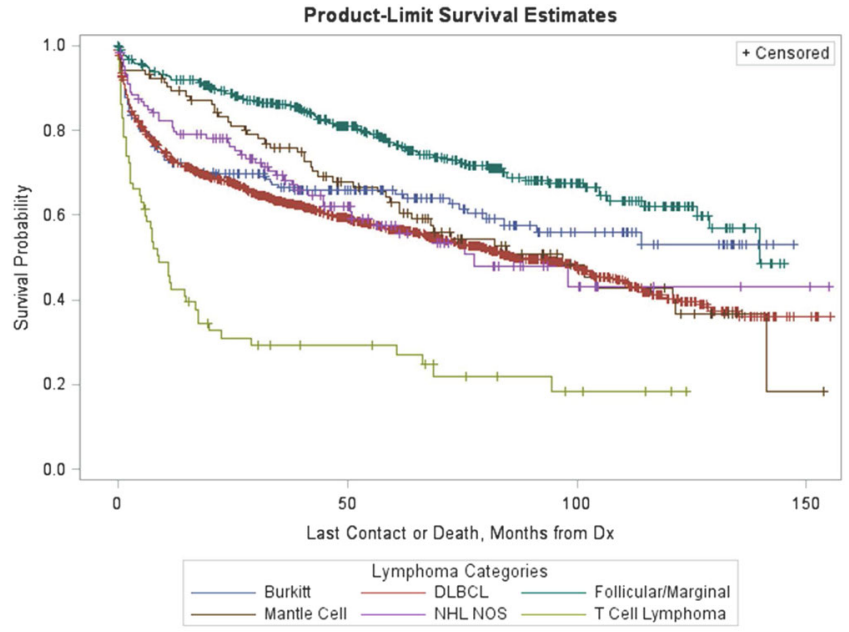

FIGURE 4 Kaplan-Meier curve for overall survival stratified by lymphoma subtype. DLBCL, diffuse large B-cell lymphoma; NHL, nonHodgkin lymphoma; NOS, not otherwise specified [Color figure can be viewed at wileyonlinelibrary.com] 


\section{4 | DISCUSSION}

We present a modern cohort of 2153 nonmetastatic surgical PCL patients undergoing nonpalliative resection. Our analyses reveal that PCL patients suffer high short-term mortality, have high rates of margin positivity, and have an overall survival which is associated with age, comorbid conditions, histologic subtype, and receipt of adjuvant chemotherapy.

The older age and male predominance observed in our cohort are similar to that reported in multiple studies across diverse patient populations., 51,12 Similarly, the preponderance of B-cell lineage tumors, in particular, DLBCL, is comparable to other reported series of $\mathrm{Gl}$ and colorectal lymphoma. ${ }^{4}$

In comparison to other large published series, our study reflects modern care patterns and focuses on nonmetastatic surgical patients. The largest study of PCL to date included 3342 patients collected from the Surveillance, Epidemiology, and End Results (SEER) database, and demonstrated improved survival among patients who underwent surgery. ${ }^{5}$ However, further survival analyses demonstrated a benefit from surgery only among early-stage patients, patients with right-sided tumors, and patients with DLBCL. Patients with indolent lymphoma, left-sided lesions, and advanced disease did not experience a survival benefit of surgery. Notably, however, more than $30 \%$ of patients in the study were treated before the year 2000, 21\% had stage IV disease, and only $60 \%$ underwent surgery.

Unlike this and other reported series of PCL, our study focused only on likely oncologically "good risk" patients, in that they received nonpalliative surgery and that stage IV patients were excluded. However, despite the exclusion criteria, analysis revealed a high rate of margin positivity and a high 30- and 90-day mortality. Only 54\% of patients achieved $\mathrm{RO}$ resection. The deidentified nature of the database makes it impossible to determine the reason for $\mathrm{R} 1$ resection, but these data have implications for surgical planning and could provide a rationale for condition of neoadjuvant therapy. RO resection was not predictive of overall survival but may have other implications for patients.

Both 30 and 90- day mortality was unexpectedly high in this analysis, comparing unfavorably to published short-term mortality rates for surgical patients with other $\mathrm{Gl}$ cancers. ${ }^{13}$ Potential contributory factors include the advanced age of the patients and comorbid conditions, although surgical and tumor-specific factors may also contribute. Although the nature of the database prohibits a complete understanding of the high mortality rate, it should be considered in operative planning and patient counseling.

Multivariable analysis identified age, Charlson score, lymphoma histologic subtype, and receipt of adjuvant chemotherapy as independent predictors of overall survival. Among these, administration of chemotherapy is the sole modifiable risk factor. Adjuvant chemotherapy has previously been shown to be favorably associated with overall survival. ${ }^{14}$ However only $39 \%$ of patients in this study received it. The low rate of adjuvant chemotherapy usage may be due to patient fitness, surgical complications, patient refusal, or failure to offer chemotherapy after resection.
The low rates of neoadjuvant therapy in our study indicate that the majority of surgical patients underwent a "surgery first" approach, whether by selected treatment strategy or surgical urgency. Not included in this study is the outcome of the heterogeneous population of patients who undergo nonoperative therapy alone. When considering surgery vs chemotherapy for a PCL patient, intestinal perforation, and hemorrhage on chemotherapy is often a major concern. Recognition that this complication is less frequently observed than previously expected ${ }^{15}$ has led to a paradigm shift in the treatment of gastric lymphoma. Successful employment of neoadjuvant chemo-radiation has diminished the role of surgery, ${ }^{16}$ allowing for organ preservation with equivalent survival outcome. ${ }^{17}$ Given the high rate of margin positivity, high operative mortality, and the low rate of adjuvant chemotherapy, consideration of up-front chemotherapy/radiation is warranted in PCL as well.

Our study has several limitations based on the number of variables available for the analysis. We were reliant on the NCDB coding of staging and metastatic disease. In addition, we lacked data on the type of chemotherapy administered and some lymphoma-specific variables such as the International Prognostic Index. As we do not have reliable data on decision-making around chemotherapy administration, the potential for selection bias exists in the overall survival analysis. It is unclear whether the benefit we observe in adjuvant therapy is related to the chemotherapy or merely the fitness to receive chemotherapy. We attempted to address this by only including patients who survived for 90 days in our survival analysis. Furthermore, the lack of coding of urgent vs elective surgeries makes it impossible to determine the role of acute lymphoma presentations.

\section{5 | CONCLUSIONS}

In summary, surgical patients with PCL who suffer high rates of shortterm mortality, are likely to have R1 or R2 resections, and are relatively unlikely to receive adjuvant therapy, although adjuvant chemotherapy provides a clear survival benefit. Prognostication of surgical patient outcome may be improved by these results: overall survival is associated with age, comorbidity score, lymphoma subtype, and administration of adjuvant chemotherapy. Despite the limitations of the analysis, these data may assist physicians in surgical planning, patient counseling, and designing further studies for this rare and morbid malignancy.

\section{ACKNOWLEDGMENT}

Presented as a poster presentation at the American Society of Colon and Rectal Surgeons, Annual Meeting, 19 to 23 May 2018, Nashville, TN

\section{CONFLICT OF INTERESTS}

The American College of Surgeons and the $\mathrm{CoC}$ have not verified and are not responsible for the analytic or statistical methodology used, or the conclusions drawn from these data by the investigators. 


\section{AUTHOR CONTRIBUTIONS}

Study conception and design: Maguire and Hawkins. Acquisition of data: Hawkins. Analysis and interpretation of data: Maguire and Hawkins. Drafting of Manuscript: Maguire and Hawkins. Critical revision: Maguire, Geiger, Hardiman, Regenbogen, Ford, Muldoon, Hopkins, and Hawkins.

\section{DATA AVAILABILITY}

Data from this manuscript will not be shared, but are available on reasonable request to the NCDB by investigators associated with $\mathrm{CoC}$ cancer programs. Data used in this analysis was obtained from the NCDB Participant Use File (PUF). The PUF contains deidentified patient-level data that do not identify hospitals, health care providers, or patients as agreed to in the Business Associate Agreement that each $\mathrm{CoC}$-accredited program has signed with the American College of Surgeons. The PUFs are designed to provide investigators associated with $\mathrm{CoC}$-accredited cancer programs with a data resource they can use to review and advance the quality of care delivered to cancer patients through analyses of cases reported to the NCDB. NCDB PUFs are only available through an application process to investigators associated with $\mathrm{CoC}$-accredited cancer programs. Per agreement with the NCDB, the authors are not allowed to make this data publicly available (https://www.facs.org/ quality-programs/cancer/ncdb/puf).

\section{ORCID}

Lillias H. Maguire (D) http://orcid.org/0000-0002-6041-2416

Karin M. Hardiman (D) http://orcid.org/0000-0001-5172-2116

Alexander T. Hawkins (D) http://orcid.org/0000-0002-8531-7247

\section{REFERENCES}

1. Fan CW, Changchien CR, Wang JY, et al. Primary colorectal Iymphoma. Dis Colon Rectum. 2000;43(9):1277-1282.

2. Swerdlow SH, Campo E, Pileri SA, et al. The 2016 revision of the World Health Organization classification of lymphoid neoplasms. Blood. 2016;127:2375-2390.

3. Kim SJ, et al. Multicenter retrospective analysis of 581 patients with primary intestinal non-hodgkin lymphoma from the Consortium for Improving Survival of Lymphoma (CISL). BMC Cancer. 2011;11(1):321.

4. Cheung MC, Housri N, Ogilvie MP, Sola JE, Koniaris LG. Surgery does not adversely affect survival in primary gastrointestinal lymphoma. J Surg Oncol. 2009;100(1):59-64.

5. Cai YB, Chen H, He JJ, et al. The role of surgical intervention in primary colorectal lymphoma: A SEER population-based analysis. Oncotarget. 2016;7(44):72263-72275.

6. Lai YL, Lin JK, Liang WY, Huang Y, Chang SC. Surgical resection combined with chemotherapy can help achieve better outcomes in patients with primary colonic lymphoma. J Surg Oncol. 2011;104(3): 265-268.

7. Freedman AS, Friedberg JW Initial treatment of limited stage diffuse large B cell lymphoma. In: A. R. Rosmarin, eds. UpToDate (2018). https://www.uptodate.com/contents/initial-treatment-oflimited-stage-diffuse-large-b-cell-lymphoma. Accessed August 9, 2018.

8. Lerro CC, Robbins AS, Phillips JL, Stewart AK. Comparison of cases captured in the national cancer data base with those in populationbased central cancer registries. Ann Surg Oncol. 2013;20(6):17591765.

9. Winchester DP, Stewart AK, Phillips JL, Ward EE. The National Cancer Data Base: past, present, and future. Ann Surg Oncol. 2010;17(1):4-7.

10. Bilimoria KY, Stewart AK, Winchester DP, Ko CY. The National Cancer Data Base: a powerful initiative to improve cancer care in the United States. Ann Surg Oncol. 2008;15(3):683-690.

11. Ge Z, Liu Z, Hu X. Anatomic distribution, clinical features, and survival data of 87 cases primary gastrointestinal lymphoma. World J Surg Oncol. 2016;14:85.

12. Kim YH, Lee JH, Yang SK, et al. Primary colon lymphoma in Korea: a KASID (Korean Association for the Study of Intestinal Diseases) study. Dig Dis Sci. 2005;50:2243-2247.

13. Damhuis RA, Wijnhoven BP, Plaisier PW, Kirkels WJ, Kranse R, vanLanschot JJ. Comparison of 30-day, 90-day and in-hospital postoperative mortality for eight different cancer types. $\mathrm{Br} J$ Surg. 2012;99(8):1149-1154.

14. Chen $Y$, Chen $Y$, Chen S, et al. Primary gastrointestinal lymphoma: a retrospective multicenter clinical study of 415 cases in Chinese province of Guangdong and a systematic review containing 5075 Chinese patients. Medicine. 2015;94(47):e2119.

15. Brincker H, D'Amore F. A retrospective analysis of treatment outcome in 106 cases of localized gastric non-Hodgkin lymphomas. Danish Lymphoma Study Group, LYFO. Leuk Lymphoma. 1995;18: 281-288.

16. Yoon SS, Coit DG, Portlock CS, Karpeh MS. The diminishing role of surgery in the treatment of gastric lymphoma. Ann Surg. 2004;240(1): 28-37.

17. Willich NA, Reinartz G, Horst EJ, et al. Operative and conservative management of primary gastric lymphoma: interim results of a German multicenter study. Int J Radiat Oncol Biol Phys. 2000;46: 895-901.

18. National Cancer Database [dataset]. Chicago, IL: American College of Surgeons. https://www.facs.org/quality-programs/cancer/ncdb/puf

\section{SUPPORTING INFORMATION}

Additional supporting information may be found online in the Supporting Information section.

How to cite this article: Maguire LH, Geiger TM, Hardiman $\mathrm{KM}$, et al. Surgical management of primary colonic lymphoma: Big data for a rare problem. J Surg Oncol. 2019;120:431-437. https://doi.org/10.1002/jso.25582 